\section{Epidermal Growth Factor Receptor Gene Copy Number in Esophageal Cancer and Outcome Prediction to Gefitinib: Does Intratumoral Heterogeneity Matter?}

To THE EDITOR: I read with great interest the recently published study by Janmaat et al, ${ }^{1}$ who reported on biologic and clinical factors associated with gefitinib therapy outcome in advanced esophageal cancer. Within a panel of candidate predictive markers, they analyzed epidermal growth factor receptor (EGFR) protein expression by immunohistochemistry (IHC) and EGFR gene copy number by chromogenic in situ hybridization (CISH). Twenty-four tumors were evaluated for EGFR expression by IHC and nine were deemed highly positive (3+) for EGFR. High-level EGFR expression was significantly associated with disease control (response + stable disease) in six of these nine cases. In contrast, analysis of EGFR copy number was performed only on 16 available tumors, of which four exhibited high EGFR expression. Two of these tumors, both with high EGFR expression, were deemed CISH positive and were eventually associated with a partial response in one of the two cases. The authors concluded that assessment of EGFR level of expression by IHC predicts outcome to gefitinib therapy more effectively than EGFR copy number by CISH. Despite the small patient cohort, this study, however, does raise a few points which may help to interpret the lack of predictive value yielded by CISH and as such warrant further discussion.

The report by Hanawa et al, ${ }^{2}$ quoted by Janmaat et al, ${ }^{1}$ found that 17 of 18 high-level EGFR expressing tumors had either EGFR amplification or low level amplification/polysomy by fluorescent in situ hybridization, thus suggesting that an increased number of gene copies influences the high protein expression in $94 \%$ of cases. This figure, among tumors with strong EGFR expression, is much higher than the prevalence that Janmaat et $\mathrm{al}^{1}$ reported in their study (50\%), although the cut offs adopted in both studies to score EGFR by in situ hybridization were similar. By mere extrapolation, this discrepancy still persists when analysis of EGFR copy number is extended to $1+$ and 2+ EGFR staining tumors, questioning the prevalence of increased EGFR copy number in esophageal cancer, and the relationship with protein expression.

In several cancer types, a correlation between increasing levels of EGFR protein expression by IHC and increased gene copy number suggests that the addictive effect of gene amplification or chromosome polysomy is a relevant mechanism underlying protein expression. Remarkably, using serial sections, it has been demonstrated that regions with EGFR amplification in esophageal cancer coincided with those exhibiting $2+$ or $3+$ degrees of immunoreactivity by IHC. ${ }^{2}$ In particular, neoplastic cells showing such immunohistochemical features were found to constitute between $10 \%$ and $90 \%$ of the total cells. ${ }^{2}$ Consistent with previous investigations, ${ }^{3}$ these findings imply that the proportion of tumor cells displaying EGFR gene increased copy number may also vary across a tumor according to the area and the section being analyzed. As a result, the significant heterogeneity inherent to EGFR genetic alterations could contribute to foci with EGFR increased copy number being overlooked, and might provide an explanation for the low prevalence of tumors with EGFR increased copy number reported by Janmaat et $\mathrm{al}^{1}$ in their study. Furthermore, these observations might explain the disagreement between the reported rates of strong EGFR protein expression and the rates of increased gene copy number, especially if assays were not performed on serial sections, as could be the case in this study. This also raises the possibility that the results obtained from tumor cross section analysis may not be representative of the whole tumor.

Though several studies evaluated EGFR gene status by fluorescent in situ hybridization or CISH in a variety of tumors, there are no definitive recommendations on how to proceed with EGFR scoring. Given the real and perceived heterogeneity of EGFR distribution in tumor sections, a score based on the mean number of EGFR copies per cell or the mean ratio of gene to chromosome copy, as it is currently recommended with respect to evaluation of HER2 gene status in breast cancer, ${ }^{4}$ still requires thorough validation. Conversely, an approach based on the percentage of cells displaying specific patterns of EGFR and chromosome 7 copy numbers has proven useful in segregating subgroups of non-small-cell lung cancer patients with different outcomes after gefitinib therapy. ${ }^{5,6}$ This kind of approach might hold promise also in evaluating response prediction to tyrosine kinase inhibitors through EGFR gene and protein assessments within heterogeneous cancers, such as esophageal cancer. Unless investigators validate their methodology taking into account intratumoral genetic heterogeneity, and unless the studies are conducted on large cohorts of patients, a problem might present itself in evaluating EGFR copy number with in situ hybridization techniques.

\section{Nicola Personeni}

Gastrointestinal Oncology Unit, Gasthuisberg University Hospital, Leuven, Belgium

\section{REFERENCES}

1. Janmaat ML, Gallegos-Ruiz MI, Rodriguez JA, et al: Predictive factors for outcome in a phase II study of gefitinib in second-line treatment of advanced esophageal cancer patients. J Clin Oncol 24:1612-1619, 2006

2. Hanawa M, Suzuki S, Dobashi $Y$, et al: EGFR protein overexpression and gene amplification in squamous cell carcinomas of the esophagus. Int $\mathrm{J}$ Cancer 118:1173-1180, 2006

3. Owonikoko T, Rees M, Gabbert HE, et al: Intratumoral genetic heterogeneity in Barrett adenocarcinoma. Am J Clin Pathol 117:558-566, 2002

4. Ellis 1O, Bartlett J, Dowsett M, et al: Best Practice No 176: Updated recommendations for HER2 testing in the UK. J Clin Pathol 57:233-237, 2004

5. Cappuzzo F, Hirsch FR, Rossi E, et al: Epidermal growth factor receptor gene and protein and gefitinib sensitivity in non-small-cell lung cancer. J Nat Cancer Inst 97:643-655, 2005

6. Hirsch FR, Varella-Garcia M, McCoy J, et al: Increased epidermal growth factor receptor gene copy number detected by fluorescence in situ hybridization associates with increased sensitivity to gefitinib in patients with bronchioloalveolar carcinoma subtypes: A Southwest Oncology Group Study. J Clin Oncol 23:6838-6845, 2005

DOI: $10.1200 / J C O .2006 .07 .5622$

Author's Disclosures of Potential Conflicts of Interest

The author indicated no potential conflicts of interest. 